\title{
A NANOCOMPOSITE BASED ON MICROBIAL SYNTHESIS OF HUMIC PRECURSORS AND OF METAL NANOPARTICLES USED AS AN ANTIMICROBIAL COMPOUND IN SOIL
}

\author{
Sorin Matei ${ }^{1}$, Gabi-Mirela Matei ${ }^{1}$, Gina Cogălniceanu ${ }^{2}$, Alexandru \\ Brînzan ${ }^{2}$
}

Key words: nanocomposite, microbial synthesis, humic precursors, microbial consortia, silver nanoparticles, antimicrobial activity

\begin{abstract}
Soil humic precursors could be considered the most active and mobile fraction and are highly significant to a series of biochemical processes in all types of soil. The microbial biosynthesized humic precursors attracted increasing attention on green synthesis of nanocomposite compounds realized between biopolymers and metal nanoparticles. Silver nanoparticles are the most used engineered nanocomposite serving as antimicrobial agents.

In the present study we used aqueous solution of humic precursors synthesized by four microbial consortia (C1-C4) and selected on the basis of high quantities of exometabolites with structural similarities to soil humic acid fraction. The humic precursors were used as capping agents of silver nanoparticles in the nanocompozite synthesis.

Biosynthesized humic precursors act as reductive and stabilizative agents of nanoparticles which are found between 5-300nm in size and with spherical preponderant shape.

The presence of humus precursors and the biosynthesized silver nanoparticles was confirmed by FTIR and UV-Vis.

At a given precursor concentration, the efficiency of nanocomposite synthesis increased with particle concentration and time of reaction, property which can be attributed to the high reduction capacity of humic precursors.

The induced antimicrobial effect of exposure to nanocomposites differs due to the size, time of preparation and stability.

Stabilization of nanocomposite by specific metal-ligand bonds was obtained in the solution for three months without any precipitate.

The antimicrobial effect of nanocomposites was estimated under laboratory agar well diffusion tests against mycotoxigenic soil fungal isolate Aspergillus niger

1 National Research-Development Institute for Soil Science, Agrochemistry and Environment, Bucharest, Romania, e-mail: so_matei602003@yahoo.com

2 Institute of Biology, Bucharest, Romania
\end{abstract}


(A27). The green synthesis of nanocomposite material with the best antimicrobial effect against test fungus was realized by microbial consortium C3and C4.

\section{Introduction}

Silver $(\mathrm{Ag})$ has a strong toxicity to a broad spectrum of microorganisms. Due to their antimicrobial properties, silver nanoparticles (AgNPs) were obtained by nanotechnologies involving reducing agents of chemical or biological nature (Sarsar et al., 2014; Elbeshehy et al., 2015; Khodashenas and Ghorbani, 2015) and used in biomedical applications, food preservation and other ecological disinfection processes (Prabhu and Poulose, 2012; Matei et al., 2015).

Humic precursors have a variety of components including quinone, phenol, catechol and sugar moieties. The functional groups which contribute most to surface charge and reactivity of prehumic substances are phenolic and carboxylic groups. The molecules form supramolecular structures held together by noncovalent forces, such as Van der Waals force, $\pi-\pi$, and CH- $\pi$ bonds.

Nanocomposites made of humic precursors and silver nanoparticles could be an alternative in production of bactericidal products (Akaighe et al., 2011). By the ratio of large surface, volume, and high active surface, metal nanoparticles have properties to influence the growth of catalytic activity.

Nanocomposites made from humic precursors biosynthesized by microbial consortia and silver nanoparticles attempt to capitalize matrix characteristics for obtaining antimicrobial nanocomposites and selecting efficient consortia. The AgNPs toxicity depends on their size and shape because the control of these parameters becomes a crucial factor during their synthesis and stabilization (Ramirez et al., 2009; Cunha et al., 2017).

Non-toxic nanocomposites with antimicrobial activity can be synthesized by reducing silver nitrate in the presence of biopolymers (Lam et al., 2018).

The nanocomposites with polyvalent and synergistic antimicrobial effects can effectively kill soil pathogenic microflora without the appearance of adaptative resistance (Xiaomei et al., 2017; 2018; Matharu et al., 2018).

The antimicrobial properties of such nanocomposites can be also exploited in products such as antimicrobial sprays, cosmetics, detergents, food supplements (Tran et al., 2013).

Research has been carried out to assess the influence of biopolymers as humic precursors from to four microbial consortia $(\mathrm{C} 1-\mathrm{C} 4)$ as a silver reducing and capping agents for synthesis of nanocomposite materials as new efficient antimicrobial agents. 


\section{Material and methods}

Microorganisms -A mycotoxigenic soil fungal isolate of Aspergillus niger (A27) obtained from laboratory collection of INCDPAPM-ICPA Buc. was used for assessing antimicrobial activity of prepared nanocomposites.

The humic precursors were biosynthesized by four soil microbial consortia C1-C4 (including 22 isolates of bacteria and fungi belonging to genera Pseudomonas, Lactobacillus, Streptomyces, Fusarium and Botrytis) selected on the basis of quantities of exometabolites and the structural similarities of compositions with standard humic acid fractions, as presented in previous published results (Matei et al., 2016).

Exometabolites were assessed by spectrophotometric method for absorbance at two wavelengths 465 and $665 \mathrm{~nm}$, with a standard working solution containing humic acid (HA) prepared in pyrophosphate alkali solvent.

Preparation of nanocomposites - The nanocomposite materials were synthesized by in situ chemical oxidation-polymerization of silver nitrate $(1 \mathrm{mM})$ with biopolymers from consortia $\mathrm{C} 1-\mathrm{C} 4$. The mixture was allowed to react for $48 \mathrm{~h}$ under constant stirring at $25^{\circ} \mathrm{C}$.

UV-Vis spectroscopy -The absorption spectrum of the reaction mixtures was recorded at room temperature using UV-Vis spectrophotometer (Rayleigh, UV2100) at a resolution of $1 \mathrm{~nm}$.

FT-IR spectroscopy -The products were analysed by Fourier transform infrared (FT-IR) spectroscopy using $\mathrm{KBr}$ disks with spectrophotometer over the 4000 to $400 \mathrm{~cm}^{-1}$ range, at a rate of $16 \mathrm{~nm} / \mathrm{s}$ (Gerasimowisz and Byler, 1985).

TEM- Transmission electron microscopy analysis of silver nanoparticles size and shape was performed using (TEM) JEM - 1400 (Jeol) microscope (80kV accelerating voltage), according to the method described by Dwivedi and Gopal (2010).

SEM-Field emission scanning electron microscopy was applied to observe the surface morphology of nanocomposite materials using a (SEM) JSPM - 5200 instrument (10kV accelerating voltage), accordingly to Paulo et al., (2012).

Antimicrobial activity assay -The antimicrobial activity of nanocomposite materials was tested against mycotoxigenic soil fungi Aspergillus niger (A27) using agar well diffusion method (Magaldi et al., 2004).

\section{Results and discussion}

Silver nanoparticle colloidal solutions were synthesized by using biopolymers as reducing agents. Since biopolymers were biosynthesized by microbial consortia $\mathrm{C} 1-\mathrm{C} 4$, the composition and reactivity were different from one to another.

Extracellular extracts with humic precursors biosynthesized by consortia C1$\mathrm{C} 4$ are presented in Figure 1. 
The biopolymers acted as reducing agents of the silver $(\mathrm{Ag}+)$ ions.

For assessing the concentration of exometabolites, similar in structure with humic acids, in this study were used aqueous solutions of standard humic acids. Results evidenced the presence and the level of biopolymers in culture medium at all four microbial consortia (C1-C4). Also, every consortium was analysed for the quantities of exometabolites produced and for similarities with compozition of humic acid subfractions.

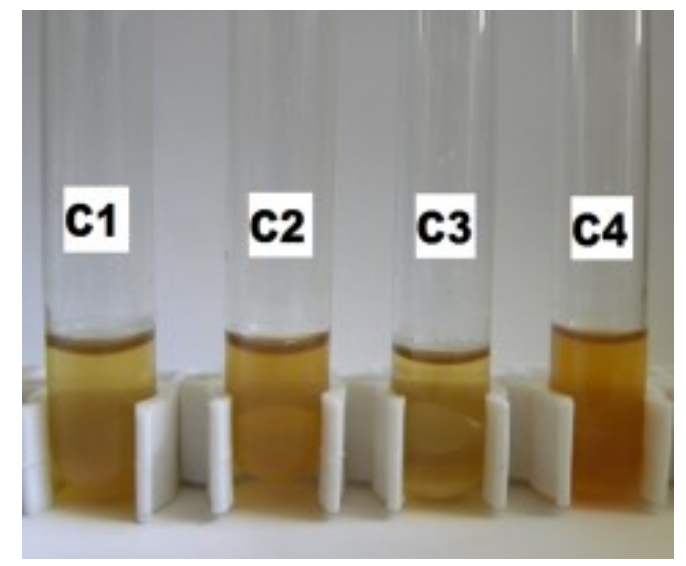

Fig. 1 Humic precursors biosynthesized by consortia C1-C4

The FTIR spectra of the biosynthesized humic precursors exhibited different absorbances due to the different qualitative compositions and the different spectra aspect was due to the relative intensity of some bands. Thus, the main bands attributions (according to Tatzber et al., 2007) comprise a broad band at 3600-3200 $\mathrm{cm}^{-1}$, which corresponds to the $\mathrm{OH}$ groups, organic phenols and acids $\mathrm{H}$-linked; a band at $2930-2920 \mathrm{~cm}^{-1}$ from the tensioning of the C-H alkyl structures; a broad band at $1650-1630 \mathrm{~cm}^{-1}$ with the first pick at $1600 \mathrm{~cm}^{-1}$ and the second less picked at $1540-1550 \mathrm{~cm}^{-1}$ produced mainly by the aromatic groups $\mathrm{C}=\mathrm{C}, \mathrm{C}=\mathrm{O}$ of amides (I), ketones and quinones; a broad band between 1120 and $980 \mathrm{~cm}^{-1}$, centered at $1034 \mathrm{~cm}^{-1}$, which generally characterize aromatic ethers as well as carbohydrates. All spectra of humic precursors showed a high intensity at the bands: 3400,1650 and $1034 \mathrm{~cm}^{-1}$.

Biosynthesized humic precursors proved to be efficient reductive and stabilizative agents and formed silver nanoparticles with $5-300 \mathrm{~nm}$ in size and with spheric preponderant shape.

The colour of reaction mixture between humic precursors and $\mathrm{AgNO}_{3}$ changed from yellow to brown after 48 hours from the addition of $\mathrm{AgNO}_{3}(1 \mathrm{mM})$ due to 
excitation of Surface plasmon vibration in metal nanoparticles (Singh and Raja, 2011).

AgNPs synthesized using humic precursors from $\mathrm{C} 1-\mathrm{C} 4$ acting as reductors of silver ions were grouped in function of the sizes, in five categories: $5-10 \mathrm{~nm} ; 10$ 30nm; 30-100nm; 100-150nm; 150-200nm (Fig. 2).

For all C1-C4 humic precursors preponderant was the group of AgNPs with the sizes $5-10 \mathrm{~nm}$. In the case of humic precursors from consortium $\mathrm{C} 4$, the presence of AgNPs in the 5-10nm group represented $71 \%$ from all the mixture (Fig. 2) and with a prevalent spheric shape (Fig. 3).

Surface plasmon resonance occurs at $420-450 \mathrm{~nm}$ as indicative of silver nanoparticles formation.

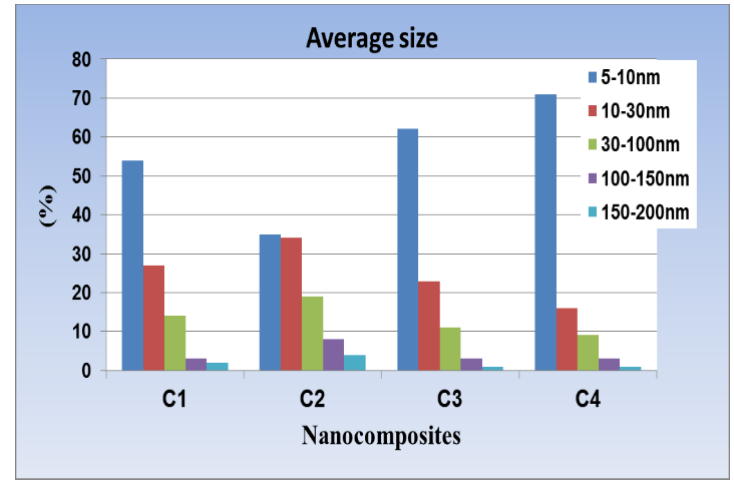

Fig. 2 Effect of reductors of consortia C1-C4 on the size of AgNPs

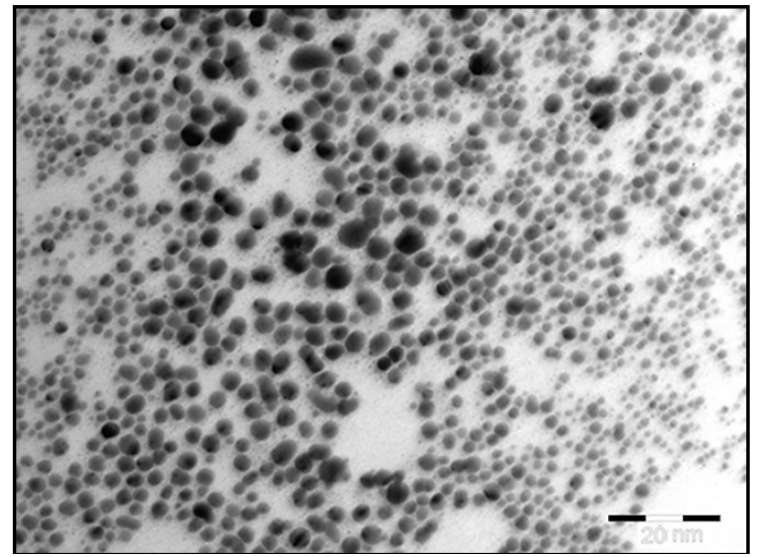

Fig. 3 TEM images of spherical AgNPs synthesized 
with humic precursors from consortium $\mathrm{C} 4$

The biosynthesis of silver nanoparticles mediated by humic precursors from consortia C1-C4 was confirmed by UV-Vis spectroscopy at 430nm (Fig. 4). The reduction process of $\mathrm{Ag}^{+}$to $\mathrm{Ag} 0$ and $\mathrm{AgNPs}$ formation was monitored by absorption and fluorescence spectral changes in addition to colour change of reaction mixture from yellow to brown. The results showed the maximum absorption peak at the wavelength of 430nm which refers to silver nanoparticles formation, according to statements from literature, that "AgNPS exhibit maximum UV-visible absorption in the range of 400-500 nm because of surface plasmon resonance" (Sestry et al., 1997; Al-Zahrani and Al-Garni, 2019).

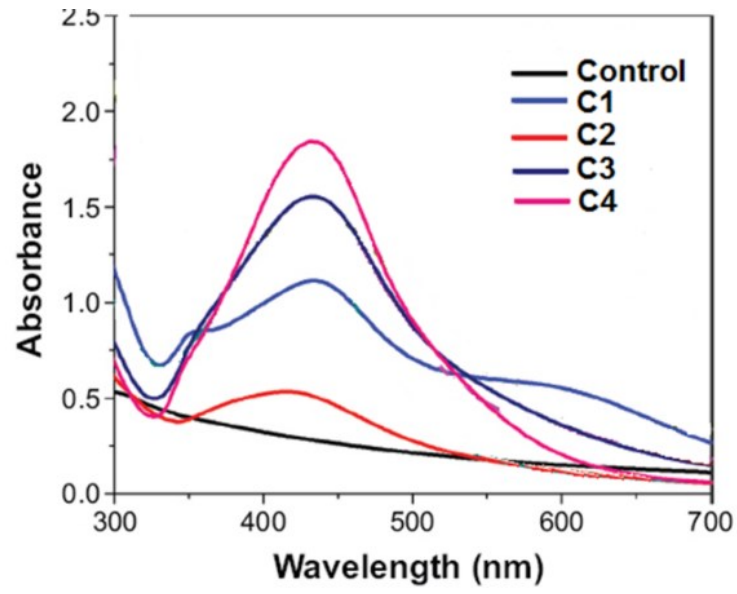

Fig. 4 UV-visible spectrum of AgNPs with humic precursors from consortia C1-C4

The humic precursors from consortia C1-C4 also acted as capping agents of silver nanoparticles in synthesis of nanocomposite materials (Fig. 5).

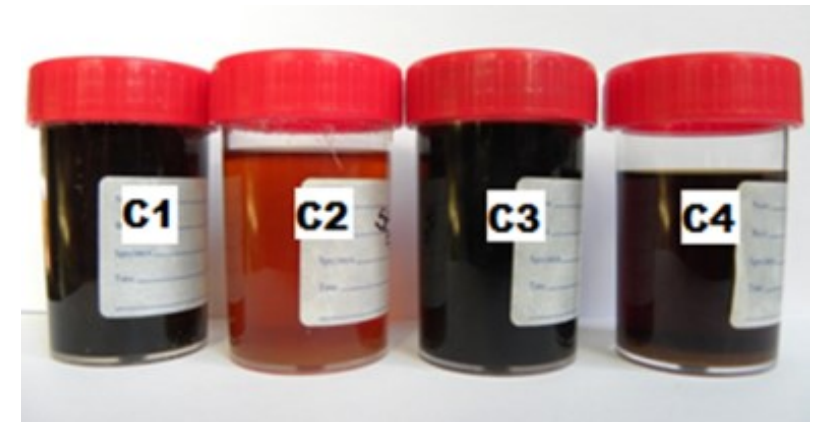

Fig. 5 Nanocomposites between humic precursors from consortia C1-C4 
and silver nanoparticles

As in a typical synthesis process, humic precursors existing in the resulted solution of colloidal Ag nanoparticles realized with these a complex mixture in aqueous solution. The mixture was allowed to react under constant stirring.

The morphology of the nanocomposite materials realized as a result of the complex mixture particularities is shown in Figure 6. These SEM images evidenced the level of porosity and structural organization of nanocomposite material which contains precursors coated silver nanoparticles.

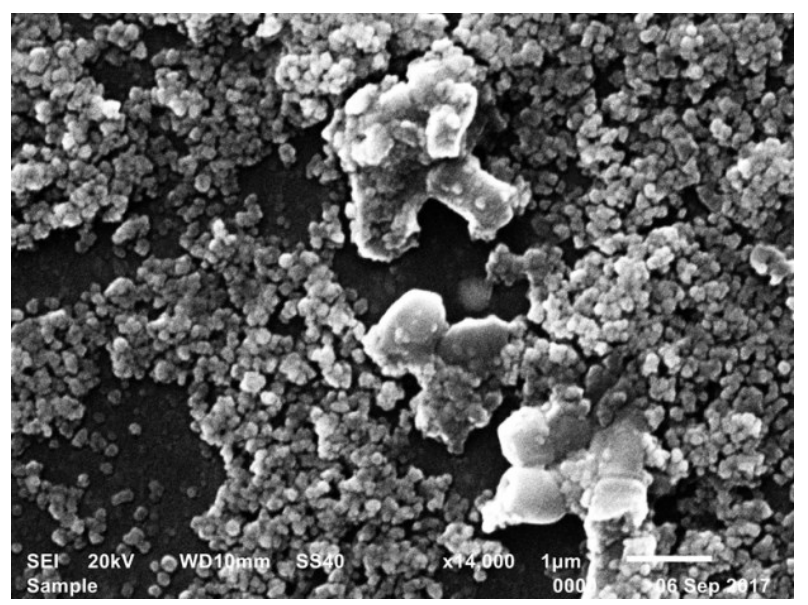

Fig. 6 SEM images of morphology of nanocomposite of consortium C3

The induced antimicrobial effect by exposure to nanocomposites differs as a function of their size, the time of preparation and stability.

The effect of nanocomposite materials on mycotoxigenic soil fungi was estimated in laboratory under the agar well diffusion tests against fungal isolate of Aspergillus niger (A27).
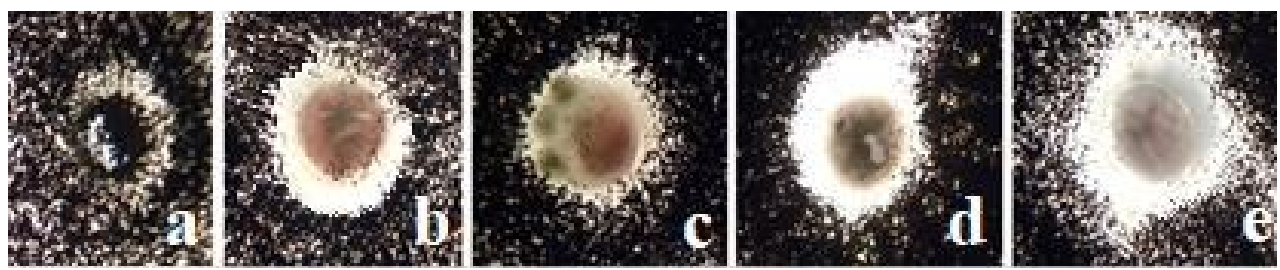

Fig. 7 Antifungal activity of nanocomposites against Aspergillus niger a) Control; b) Consortium $\mathrm{C} 1$; c) Consortium C2; d) Consortium C3; e) Consortium C4; 
The green synthesized nanocomposites revealed the best antimicrobial effect against fungal isolate in the case of microbial consortia $\mathrm{C} 3$ and $\mathrm{C} 4$. The average formed inhibition zone was of $12 \mathrm{~mm}$ for $\mathrm{C} 3$ and $15 \mathrm{~mm}$ for $\mathrm{C} 4$ (Fig. 7 and 8).

The results are similar to those reported for uniform spherical AgNPs of $1-10 \mathrm{~nm}$ diameter (obtained using fruit waste material as reducing agent) that presented antimicrobial activity against Salmonella sp., Proteus sp., Klebsiella sp., Candida sp. and Aspergillus sp. (Kirunthika and Somanathan, 2014).

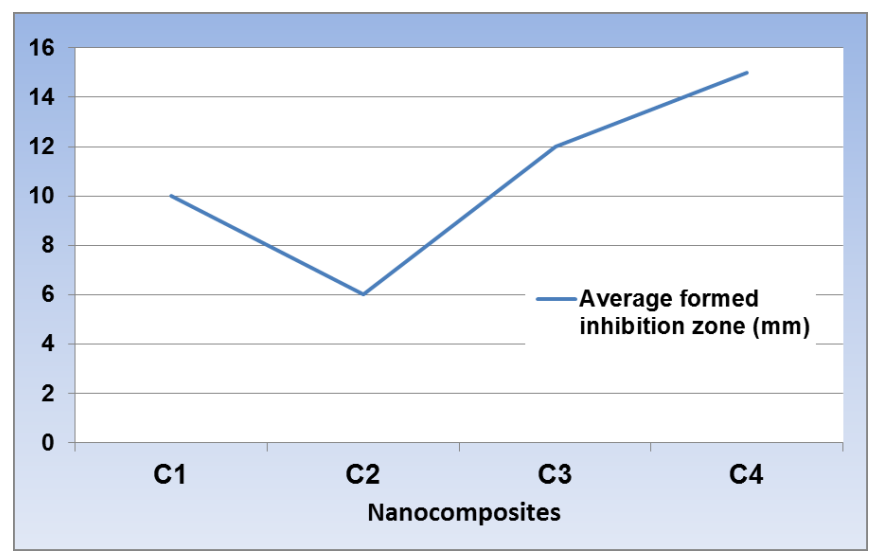

Fig. 7 Average of inhibition zones obtained from nanocomposites C1-C4 against mycotoxigenic fungus Aspergillus niger (A27)

The stability of nanocomposites was maintained in the solution for three months without any precipitate by the specific metal-precursors bonds.

\section{Conclusion}

The synthesis of silver nanoparticles under the consortia C1-C4 biopolymers was confirmed at $430 \mathrm{~nm}$.

AgNPs obtained had between 5-300nm in sizes and a spherical preponderant shape under the reductive and stabilizative humic precursors from consortia C1-C4.

Preponderant AgNPs synthesized using humic precursors from all consortia belonged to the size group of 5-10nm.

The percentage of the AgNPs presence in 5-10nm group was of $71 \%$ under the influence of humic precursors from consortium $\mathrm{C} 4$.

The SEM morphology of nanocomposites showed the porous structure of coated nanoparticles. 
FTIR spectra of humic precursors of consortia C1-C4 showed a high intensity for bands 3400, 1650 and $1034 \mathrm{~cm}^{-1}$.

The green synthesized nanocomposites with the best antimicrobial effect against mycotoxigenic fungal isolate Aspergillus niger (A27) were those realized with compounds produced by microbial consortia $\mathrm{C} 4$ and $\mathrm{C} 3$ with high average diameter of inhibition zones.

Acknowledgments: This paper was published with financial support of National Authority for Scientific Research, under the frame of Project PN19 3404 01/2019.

\section{References:}

1. Akaighe, N., MacCuspie, R.I., Navarro, D.A., Aga, D.S., Banerjee, S., Sohn, M. Sharma, V.K., (2011), Humic acid-induced silver nanoparticle formation under environmentally relevant conditions, Environmental Sciences and Technology, 45: 3895-3901. https://doi.org/10.1021/es103946g

2. Al-Zahrani S.S., Al-Garni S.M., (2019) Biosynthesis of Silver Nanoparticles from Allium ampeloprasum Leaves Extract and Its Antifungal Activity, Journal of Biomaterials and Nanobiotechnology, 10(01):11-25, DOI: 10.4236/jbnb.2019.101002

3. Cunha M.N.M., Felgueiras H.P., Gouveia I., Zille A. (2017), Synergistically enhanced stability of laccase immobilized on synthesized silver nanoparticles with water-soluble polymers, Colloids and Surfaces B: Biointerfaces, 154: 210-220. https://doi.org/10.1016/j.colsurfb.2017.03.023

4. Dwivedi A.D., Gopal K., (2010), Biosynthesis of silver and gold nanoparticles using Chenopodium album leaf extract, Colloid. Surface A., 369(1-3): 27-33. https:// doi.org/10.1016/j.colsurfa.2010.07.020

5. Elbeshehy E., Ellazzazy A., Aggelis G., (2015), Silver nanoparticles synthesis mediated by new isolates of Bacillus spp., nanoparticle characterization and their activity against Bean Yellow Mosaic Virus and human pathogens, Frontiers in Microbiology, http://dx.doi.org/10.3389/fmicb.2015.00453

6. Gerasimowisz W.V., Byler D.M., (1985), Carbon-13 CPMAS NMR and FTIR spectroscopic studies of humic acids, Soil Science, 139: 270-278.

7. Khodashenas B., Ghorbani H.R., (2015), Synthesis of silver nanoparticles with different shapes, Arabian Journal of Chemistry, 12(8): 1823-1838 https://doi.org/10.1016/j.arabjc.2014.12.014

8. Kirunthika N., Somanathan T., (2014), Eco-synthesis of silver nanoparticles and its use to antebacterial and antifungal activity, J. of Chemical and Pharmaceutical Sciences, special issue 4: 74-76.

9. Lam S., Wong E., Boyer C., Qiao G., (2018), Antimicrobial polymeric nanoparticles, Progress in Polymer Science, 76:40-64. https://doi.org/10.1016/j.progpolymsci.2017.07.007

10. Magaldi S., Mata-Essayag S., Hartung de Capriles C., et al., (2004), Well diffusion for antifungal susceptibility testing, International Journal of Infectious Diseases, 8: 39-45.

11. Matei A., Cornea C. P., Matei S., Matei G.M., Cogălniceanu G., Rodino S., (2015), Biosynthesis of silver nanoparticles using culture filtrates of lactic acid bacteria and 
analysis of antifungal activity, Digest Journal of Nanomaterials and Biostructures, 10(4):1201-1207, http://www.chalcogen.ro/1201_Matei.pdf

12. Matei G.M., Matei S., Mocanu V., Dumitru S., Ignat P. (2016), Microbiological characterization of suppressive forest soil from Enisala, Analele Universității Craiova, Agricultură - Montanologie - Cadastru, 56:341-347.

13. Matharu R.K., Ciric L., Edirisinghe M. (2018), Nanocomposites: suitable alternatives as antimicrobial agents, Nanotechnology, 29 (28): 282001.

14. Paulo E.M., Vasconcelos M.P., Oliveira I.S., Affe H.M.J., Nascimento R., Melo I.S., Roque M.R.A., Assis S.A., (2012), An alternative method for screening lactic acid bacteria for the production of exopolysaccharides with rapid confirmation, Food Science and Technology, 32(4):710-714. https://doi.org/10.1590/S0101-20612012005000094

15. Prabhu S., Poulose E.K., (2012), Silver nanoparticles: mechanism of antimicrobial action, synthesis, medical applications and toxicity effects, International Nano Letters, 2, 32 (2012). https://doi.org/10.1186/2228-5326-2-32

16. Ramirez I.M., Bashir S., Luo Z., Liu J.L., (2009), Green synthesis and characterization of polymer-stabilized silver nanoparticles, Colloids and Surfaces B: Biointerfaces, 73 (2): 185-19. https://doi.org/10.1016/j.colsurfb.2009.05.015

17. Sarsar V., Selwal K.K, Selwal M.K. (2014), Nanosilver: Potent antimicrobial agent and its biosynthesis, African Journal of Biotechnology, vol.13, nr.4, p. 546-554. DOI: $10.5897 / A J B 2013.13147$

18. Sastry M., Mayya K.S., Bandyopadhyay K., (1997), pH Dependent Changes in the Optical Properties of Carboxylic Acid Derivated Silver Coloidal Particles, Colloids and Surfaces, A, 127:221-228.

19. Singh P., Raja R.B., (2011), Biological synthesis and characterization of silver nanoparticles using the fungus Trichoderma harzianum, Asian Journal of Experimental Biological Sciences, 2 (4): 600-605.

20. Tatzber M., Stemmer M., Spiegel H., Katzlberger C., Haberhauer G., Mentler A., Gerzabek M., (2007), FTIR-spectroscopic characterization of humic acids and humin fractions obtained by advanced $\mathrm{NaOH}, \mathrm{Na} 4 \mathrm{P} 2 \mathrm{O} 7$, and $\mathrm{Na} 2 \mathrm{CO} 3$ extraction procedures. Journal of Plant Nutrition and Soil Science 170(4):522-529. https://doi.org/10.1002/jpln.200622082

21. Tran Q.H., Nguyen V.Q., Le A.T, (2013), Silver nanoparticles: synthesis, properties, toxicology, applications and perspectives, Advances in Natural Sciences: Nanoscience and Nanotechnology, available online at: http://iopscience.iop.org/2043$6262 / 4 / 3 / 033001$

22. Xiaomei D., Xuelei C., Jing Z., Yu Z., Qianqian G., Tianqi Z., Chunli C., Xinge Z., Chaoxing L., (2017), Structure-Activity Relationship of Membrane-Targeting Cationic Ligands on a Silver Nanoparticle Surface in an Antibiotic-Resistant Antibacterial and Antibiofilm Activity Assay, ACS Applied Materials \& Interfaces, 9 (16): 13837-13848. https://doi.org/10.1021/acsami.6b15821

23. Xiaomei D., Yu Z., Junsheng L., Sen L., Ruidong L., Xuelei C., Xinge Z., Chaoxing L., (2018), Thiazolium-derivative functionalized silver nanocomposites for suppressing bacterial resistance and eradicating biofilms, New Journal of Chemistry, 42 (2): 1316-1325.

C 2020 by the authors. Licensee UAIC, Iasi, Romania. This article is an open access article distributed under the terms and conditions of the Creative Commons Attribution (CC BY-NC-ND) license (https:// creativecommons.org/licenses/by-nc-nd/4.0). 